\title{
Carcinoma of the duodenum
}

\author{
Angela Carragher and Colin Russell
}

Royal Victoria Hospital, Grosvenor Road, Belfast BT12 6BA, Northern Ireland.

\begin{abstract}
Summary: We report two patients with primary adenocarcinoma of the duodenum who underwent successful pancreatico-duodenectomy (modified Whipple operation). One patient died from an unrelated cause three years following surgery whilst the other remains well and clinically free of disease 3 years after resection. An aggressive surgical policy in patients with this rare tumour seems justified.
\end{abstract}

\section{Introduction}

Primary adenocarcinoma of the duodenum is rare. Even in a specialist referral centre, few cases are likely to be encountered during the clinical life-span of any one individual physician. Many published reports provide little cause for optimism in terms of operability and potential cure or indeed of significant survival in patients with these tumours. Others, however, engender a somewhat more hopeful outlook by reporting medium and even occasional long term survival following a radical surgical approach. We record two further patients with primary duodenal carcinoma, both of whom underwent successful pancreatico-duodenectomy.

\section{Case reports}

\section{Case 1}

A 66 year old male presented with a two month history of anorexia, $9 \mathrm{~kg}$ weight loss, severe epigastric pain and associated vomiting. Barium meal examination demonstrated a shouldered stricture $6 \mathrm{~cm}$ in length with central ulceration of the mucosa in the second part of the duodenum. At endoscopy the presence of an ulcerating tumour of the second part of the duodenum was confirmed. Biopsy showed a well differentiated adenocarcinoma. At laparotomy a small mobile supra-ampullary carcinoma of the duodenum was found. The liver was clear of metastases. Pancreatico-duodenectomy (modified Whipple's procedure) was carried out. The post-operative course was uneventful. Histopathological examination of the specimen confirmed the presence of a

Correspondence: C. Russell, F.R.C.S.

Accepted: 9 April 1987 primary adenocarcinoma of the duodenum. The patient remained well and clinically free of disease during a three year period of review. He then presented with an acute abdomen and at laparotomy was found to have infarcted his entire small bowel as a result of superior mesenteric artery thrombosis. There was no evidence of recurrent tumour locally or metastatic deposits in the liver. The patient died a few hours following operation.

\section{Case 2}

A 67 year old male presented with a one month history of crampy abdominal pain, anorexia and weight loss of $4 \mathrm{~kg}$. Examination revealed a patient who was clinically anaemic with a large, firm, smooth mass palpable in the right peri-umbilical region. His haemoglobin was $7.8 \mathrm{~g} / \mathrm{dl}$. Ultrasound examination and computed tomography confirmed the presence of a mass measuring $10 \mathrm{~cm} \times 5 \mathrm{~cm} \times 5 \mathrm{~cm}$ lying in the retroperitoneum at the inferior aspect of the head of the pancreas and in front of the inferior vena cava. Neither barium meal examination nor upper gastrointestinal endoscopy was performed. At laparotomy a large mobile mass was found in the right upper abdomen. Assessment revealed this to be a tumour lying within the infra-ampullary portion of the second part and proximal segment of the third part of the duodenum and also extending into the transverse mesocolon. Trial dissection confirmed operability and pancreatico-duodenectomy was performed. Division of the right and middle colic vessels necessitated concomitant extended right hemicolectomy. Histopathology reported a poorly differentiated primary adenocarcinoma of the duodenum with metastatic involvement of multiple adjacent lymph nodes. The patient remains alive, well and clinically free of tumour 3 years following surgery. 


\section{Discussion}

The estimated incidence of primary adenocarcinoma of the duodenum, based on autopsy studies is $0.035 \% .^{1}$ As an entity it comprises approximately $0.33 \%$ of all gastrointestinal tumours. Publications are mainly single case reports and there are few large collected series. Due to its infrequent occurrence it is difficult for any one surgeon to accrue experience in the management of this rare malignancy.

Bremer \& Brown ${ }^{2}$ describe four symptom complexes, namely (1) duodenal obstruction (2) ulceration with haemorrhage; (3) penetration causing pain and (4) biliary obstruction.

Barium meal and upper gastrointestinal endoscopy and biopsy are the mainstays of pre-operative diagnosis. It is essential when performing barium meal examination in the anaemic patient, to ensure that the entire duodenum is outlined by contrast and that screening is carried out at least as far as the duodenojejunal flexure.

Most of the reported cases of adenocarcinoma of the duodenum preceded the widespread availability of endoscopy. In the early 1970 s it was anticipated that fibreoptic endoscopy would facilitate earlier diagnosis of duodenal malignancy and, hopefully, improve both the resectability rate and the five year survival. ${ }^{3}$ In fact, apart from our first case, Swift reports the only patient from a British centre in whom a firm pre-operative histological diagnosis of primary adenocarcinoma of the duodenum was made ${ }^{4}$ during a period of approximately 15 years since the advent of fibreoscopy.

Supra- and infra-ampullary lesions are found in equal numbers. The more distally placed tumours are more likely to be amenable to local resection with direct duodeno-jejunal anastomosis. In contrast,

\section{References}

1. Kleinerman, J., Yardumian, K. \& Tamaki, H.T. Primary carcinoma of the duodenum. Ann Intern Med 1950, 32: 451.

2. Brenner, R.L. \& Brown, C.H. Primary carcinoma of the duodenum. Gastroenterology 1955, 29: 189-198.

3. Satake, K., Sowa, M., Yamashita, K. et al. Carcinoma of the duodenum: its pre-operative diagnosis. Br J Surg 1975, 62: 973-976.

4. Swift, A.C., Smith, G.T. \& Douglas, D.L. Dual primary adenocarcinoma of the duodenum and jejunum in a supra-ampullary lesions, if operable, invariably $\frac{3}{\infty}$ require more radical surgery, usually pancreaticoduodenectomy. If, however, a trial dissection demonstrates that a Whipple's operation is not feasible, a palliative by-pass procedure is indicated.

In a 20 year review of the experience accumulated in managing duodenal tumours at their institution, Lillemoe \& Imbembo report curative resections having been undertaken in six of 12 patients with primary adenocarcinoma. ${ }^{5}$ Of these, four individuals underwent pancreatico-duodenectomy with one death in the post-operative period. Alwmark and colleagues report 66 patients with duodenal carcinoma recorded by the Swedish Cancer Register over a 16 year period. ${ }^{6}$ A total of 12 individuals had a Whipple's procedure $\exists$ performed with an operative mortality rate of $17 \%$. Interestingly, of seven patients who presumably had $\omega$ distally situated tumours and who underwent local $f$ duodenal resection, two $(29 \%)$ died perioperauvely. $c$ Support for an aggressive surgical approach, when $\bigcirc$ possible, in the individual with primary adenocarcin- 은 oma of the duodenum is also reflected in the report of a large series of patients from the Mayo Clinic, ${ }^{7} 104$ patients presented over a 40 year period. Of the 53 individuals who were operated on for cure, 40 underwent radical resection with an operative mortality of $25 \%$. It is noteworthy, however, that only on operative death occurred during the latter 10 years of. the study. Further, in the patients who survived surgery a five year survival of $58 \%$ was achieved. These relatively optimistic data, coupled with our own recent but very limited experience, should, we feel, encourage the adoption of a positive and, where appropriate, radical surgical approach in the form of pancreatico-duodenectomy in the patient with primary adenocarcinoma of the duodenum.

patient with previous colonic cancers. Postgrad Med $J$ 1980, 56: 871-874.

5. Lillemoe, K. \& Imbembo, A.L. Malignant neoplasms of the duodenum. Surg Gynec Obstet 1980, 150: 822-826.

6. Alwmark, A., Anderson, A. \& Lasson, A. Primary carcinoma of the duodenum. Ann Surg 1980, 191: 13-18.

7. Joesting, D.R., Beart, R.W., van Heerden, J.A. \& Weiland, L.H. Improving survival in adenocarcinoma of the duodenum. Am J Surg 1981, 141: 228-231. 